\title{
Computational Approach to the Rational Design of Molecularly Imprinted Polymers for Voltammetric Sensing of Homovanillic Acid
}

\author{
Yolanda Diñeiro, M. Isabel Menéndez, M. Carmen Blanco-López, M. Jesús Lobo-Castañón, \\ Arturo J. Miranda-Ordieres and Paulino Tuñón-Blanco. \\ Departamento de Química-Física y Analítica, Universidad de Oviedo, C/ Julián Clavería 8, \\ Oviedo 33006, Spain.
}

\section{Supporting Information}

Contents:

Table S1: Cartesian coordinates for reactants and the most stable pre-polymerization complexes.

Table S2. Absolute energies calculated with BS I and BS II (a.u.) and ZPVE obtained with BS I (kcal/mol) for isolated reactants and all the complexes located.

Figure S1: B3LYP/6-31G* geometries of the least stable pre-polymerization complexes formed between HVA and the functional monomers MAA and VPY. 


\section{Table S1. Cartesian coordinates for reactants and the most stable pre-polymerization complexes (to be read in columns sheet by sheet)}

\begin{tabular}{|c|c|c|c|}
\hline \multicolumn{4}{|c|}{ Compound $=$ HVA } \\
\hline $\mathrm{C}$ & -0.687125 & -0.681289 & 0.516978 \\
\hline $\mathrm{C}$ & -0.138984 & -1.942269 & 0.287457 \\
\hline $\mathrm{C}$ & 1.210593 & -2.086855 & -0.052186 \\
\hline $\mathrm{C}$ & 2.021033 & -0.965414 & -0.170463 \\
\hline $\mathrm{C}$ & 1.470515 & 0.314620 & 0.054430 \\
\hline $\mathrm{C}$ & 0.131557 & 0.456040 & 0.395567 \\
\hline $\mathrm{C}$ & -2.150618 & -0.524643 & 0.905235 \\
\hline $\mathrm{C}$ & -2.898326 & 0.347623 & -0.085806 \\
\hline $\mathrm{O}$ & -2.835093 & 1.557675 & -0.138291 \\
\hline $\mathrm{O}$ & -3.643302 & -0.380458 & -0.951152 \\
\hline $\mathrm{H}$ & -4.050714 & 0.261611 & -1.563526 \\
\hline $\mathrm{H}$ & -2.629762 & -1.504507 & 0.967244 \\
\hline $\mathrm{H}$ & -2.236642 & -0.035329 & 1.882589 \\
\hline $\mathrm{H}$ & -0.763494 & -2.827272 & 0.374563 \\
\hline $\mathrm{H}$ & 1.645276 & -3.065320 & 0.230615 \\
\hline $\mathrm{O}$ & 3.336315 & -1.094533 & -0.502105 \\
\hline $\mathrm{H}$ & 3.707739 & -0.195321 & -0.533887 \\
\hline $\mathrm{O}$ & 2.376806 & 1.336629 & -0.098939 \\
\hline $\mathrm{C}$ & 1.921994 & 2.668893 & 0.092170 \\
\hline $\mathrm{H}$ & 2.786633 & 3.311332 & -0.083399 \\
\hline $\mathrm{H}$ & 1.555563 & 2.820152 & 1.115370 \\
\hline $\mathrm{H}$ & 1.125423 & 2.922577 & -0.618224 \\
\hline $\mathrm{H}$ & -0.301666 & 1.437343 & 0.553487 \\
\hline
\end{tabular}

Compound $=$ MAA

$\begin{array}{lrcc}\mathrm{C} & 0.000000 & 0.000000 & 0.000000 \\ \mathrm{C} & 0.000000 & 0.000000 & 1.495580 \\ \mathrm{C} & 1.193177 & 0.000000 & 2.102380 \\ \mathrm{C} & -1.318352 & 0.000230 & 2.225482 \\ \mathrm{O} & 0.981674 & -0.001062 & -0.714748 \\ \mathrm{O} & -1.260132 & 0.001342 & -0.510867 \\ \mathrm{H} & -1.150415 & 0.001225 & -1.480006 \\ \mathrm{H} & 2.108853 & -0.000065 & 1.520198 \\ \mathrm{H} & 1.278671 & 0.000049 & 3.185301 \\ \mathrm{H} & -1.159928 & -0.000874 & 3.307869 \\ \mathrm{H} & -1.917790 & 0.879418 & 1.962751 \\ \mathrm{H} & -1.919067 & -0.877564 & 1.961094\end{array}$

Compound VPY

$\begin{array}{llll}\mathrm{N} & 0.000000 & 0.000000 & 0.000000 \\ \mathrm{C} & 0.000000 & 0.000000 & 1.341845 \\ \mathrm{C} & 1.150029 & 0.000000 & 2.124197 \\ \mathrm{C} & 2.408560 & 0.000192 & 1.499237 \\ \mathrm{C} & 2.409075 & 0.000243 & 0.096444 \\ \mathrm{C} & 1.198017 & 0.000062 & -0.594779 \\ \mathrm{H} & -0.979890 & -0.000198 & 1.816809 \\ \mathrm{H} & 1.060984 & -0.000132 & 3.206121 \\ \mathrm{C} & 3.687371 & 0.000071 & 2.227535 \\ \mathrm{C} & 3.857576 & -0.000960 & 3.555087 \\ \mathrm{H} & 3.027685 & -0.001866 & 4.256399 \\ \mathrm{H} & 4.852382 & -0.000930 & 3.989707 \\ \mathrm{H} & 4.572512 & 0.000771 & 1.592105 \\ \mathrm{H} & 3.347531 & 0.000389 & -0.452275 \\ \mathrm{H} & 1.192181 & 0.000183 & -1.683874\end{array}$

Compound $=$ HVA-MAA

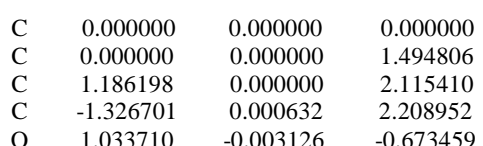

$\begin{array}{lrrr}\mathrm{O} & -1.217436 & 0.003916 & -0.524288 \\ \mathrm{H} & -1.157215 & 0.007549 & -1.527120 \\ \mathrm{H} & 2.110064 & -0.000342 & 1.546755 \\ \mathrm{H} & 1.257943 & 0.000534 & 3.199309 \\ \mathrm{H} & -1.181356 & 0.000312 & 3.293066 \\ \mathrm{H} & -1.922775 & 0.878952 & 1.936739 \\ \mathrm{H} & -1.923488 & -0.877194 & 1.936577 \\ \mathrm{O} & -1.101639 & 0.015988 & -3.205407 \\ \mathrm{C} & -0.064175 & -0.022085 & -3.870539 \\ \mathrm{O} & 1.149657 & -0.036309 & -3.353453 \\ \mathrm{H} & 1.098096 & -0.025304 & -2.349516 \\ \mathrm{C} & -0.087170 & -0.089314 & -5.385244 \\ \mathrm{H} & 0.873918 & 0.255246 & -5.773710 \\ \mathrm{H} & -0.869322 & 0.598471 & -5.726187 \\ \mathrm{C} & -0.372343 & -1.502922 & -5.871947 \\ \mathrm{C} & 0.575878 & -2.211307 & -6.608653 \\ \mathrm{C} & 0.308800 & -3.506829 & -7.063965 \\ \mathrm{C} & -0.911009 & -4.106105 & -6.778360 \\ \mathrm{C} & -1.872613 & -3.397354 & -6.026770 \\ \mathrm{C} & -1.607245 & -2.109481 & -5.579038 \\ \mathrm{H} & -2.336780 & -1.565976 & -4.989143 \\ \mathrm{O} & -3.031933 & -4.104327 & -5.812307 \\ \mathrm{C} & -4.055904 & -3.493032 & -5.040874 \\ \mathrm{H} & -4.418125 & -2.573906 & -5.518612 \\ \mathrm{H} & -4.868608 & -4.219506 & -4.987191 \\ \mathrm{H} & -3.704352 & -3.259705 & -4.028057 \\ \mathrm{O} & -1.181366 & -5.367040 & -7.217874 \\ \mathrm{H} & -2.075962 & -5.588484 & -6.904631 \\ \mathrm{H} & 1.040587 & -4.063608 & -7.640697 \\ \mathrm{H} & 1.535120 & -1.754304 & -6.835861\end{array}$

Compound $=$ HVA-VPY

$\begin{array}{lccc}\mathrm{C} & 0.000000 & 0.000000 & 0.000000 \\ \mathrm{C} & 0.000000 & 0.000000 & 1.406890 \\ \mathrm{C} & 1.220864 & 0.000000 & 2.081705 \\ \mathrm{C} & 2.431877 & -0.006005 & 1.381267 \\ \mathrm{C} & 2.431082 & -0.008376 & -0.007251 \\ \mathrm{C} & 1.201225 & -0.003730 & -0.697718 \\ \mathrm{C} & -1.313237 & 0.006108 & 2.172923 \\ \mathrm{C} & -2.051340 & 1.333372 & 2.044414 \\ \mathrm{O} & -2.029792 & 2.202518 & 2.898262 \\ \mathrm{O} & 3.605604 & -0.015620 & -0.701300 \\ \mathrm{O} & 1.340128 & -0.004836 & -2.067694 \\ \mathrm{C} & 0.161836 & 0.023364 & -2.857610 \\ \mathrm{O} & -2.706813 & 1.434891 & 0.883026 \\ \mathrm{~N} & -3.910814 & 3.932436 & 0.731033 \\ \mathrm{C} & -4.656935 & 4.430946 & -0.262790 \\ \mathrm{C} & -5.210421 & 5.706704 & -0.230254 \\ \mathrm{C} & -4.993296 & 6.532589 & 0.884493 \\ \mathrm{C} & -4.208392 & 5.998681 & 1.920460 \\ \mathrm{C} & -3.694812 & 4.712889 & 1.804549 \\ \mathrm{C} & -5.582380 & 7.880598 & 0.912493 \\ \mathrm{C} & -5.485397 & 8.776712 & 1.901786 \\ \mathrm{H} & 1.234826 & 0.004186 & 3.168198 \\ \mathrm{H} & 3.384455 & -0.008379 & 1.901872 \\ \mathrm{H} & -0.946764 & 0.019922 & -0.527620 \\ \mathrm{H} & 3.371612 & -0.009960 & -1.645945 \\ \mathrm{H} & -1.125465 & -0.160171 & 3.236068 \\ \mathrm{H} & -1.971304 & -0.790881 & 1.806708 \\ \mathrm{H} & 0.493877 & 0.026851 & -3.897531 \\ \mathrm{H} & -0.460703 & -0.862185 & -2.675963 \\ \mathrm{H} & -0.429345 & 0.926174 & -2.658077 \\ \mathrm{H} & -3.144307 & 2.342093 & 0.823674 \\ \mathrm{H} & -4.816542 & 3.779346 & -1.119129 \\ \mathrm{H} & -5.808291 & 6.057864 & -1.066734 \\ \mathrm{H} & -3.993167 & 6.574349 & 2.814674 \\ \mathrm{H} & -3.084654 & 4.263300 & 2.583633\end{array}$

$\begin{array}{llll}\mathrm{H} & -6.151730 & 8.147772 & 0.022970 \\ \mathrm{H} & -5.961434 & 9.748962 & 1.821197 \\ \mathrm{H} & -4.937969 & 8.585078 & 2.820489\end{array}$

Compound $=$ HVA $-(\text { MAA })_{2}$

\begin{tabular}{|c|c|c|c|}
\hline $\mathrm{C}$ & -0.35423 & 2.01264 & 0.68411 \\
\hline $\mathrm{C}$ & 0.07559 & 2.95937 & -0.25306 \\
\hline $\mathrm{C}$ & 1.17679 & 2.71717 & -1.07031 \\
\hline $\mathrm{C}$ & 1.8824 & 1.51109 & -0.9824 \\
\hline $\mathrm{C}$ & 1.42792 & 0.54997 & -0.0598 \\
\hline $\mathrm{C}$ & 0.33626 & 0.79757 & 0.76399 \\
\hline $\mathrm{O}$ & 2.93967 & 1.31515 & -1.80198 \\
\hline $\mathrm{O}$ & 2.09713 & -0.6719 & 0.0478 \\
\hline $\mathrm{C}$ & 1.75015 & -1.62653 & -0.9728 \\
\hline $\mathrm{C}$ & -1.55575 & 2.27749 & 1.57761 \\
\hline $\mathrm{C}$ & -2.73991 & 1.43141 & 1.149 \\
\hline $\mathrm{O}$ & -2.86011 & 0.25641 & 1.49855 \\
\hline $\mathrm{O}$ & -3.58295 & 2.0642 & 0.35435 \\
\hline $\mathrm{H}$ & -0.45211 & 3.90573 & -0.34008 \\
\hline $\mathrm{H}$ & 1.52045 & 3.45878 & -1.78458 \\
\hline $\mathrm{H}$ & 0.02305 & 0.02502 & 1.46038 \\
\hline $\mathrm{H}$ & 3.54373 & 0.61048 & -1.46988 \\
\hline $\mathrm{H}$ & 2.35278 & -2.51638 & -0.77967 \\
\hline $\mathrm{H}$ & 0.68596 & -1.87466 & -0.90389 \\
\hline $\mathrm{H}$ & 1.9788 & -1.2325 & -1.9679 \\
\hline $\mathrm{H}$ & -1.83524 & 3.33301 & 1.5333 \\
\hline $\mathrm{H}$ & -1.328 & 2.01652 & 2.61617 \\
\hline $\mathrm{H}$ & -4.31942 & 1.44442 & 0.06469 \\
\hline $\mathrm{O}$ & 4.61271 & -1.10296 & 0.97008 \\
\hline $\mathrm{C}$ & 5.42668 & -0.83195 & -0.05286 \\
\hline $\mathrm{O}$ & 5.03463 & -0.35292 & -1.11359 \\
\hline $\mathrm{C}$ & 6.85333 & -1.17756 & 0.22873 \\
\hline $\mathrm{C}$ & 7.74984 & -0.9258 & -0.73316 \\
\hline $\mathrm{C}$ & 7.19648 & -1.781 & 1.56605 \\
\hline $\mathrm{H}$ & 3.68306 & -0.8727 & 0.69993 \\
\hline $\mathrm{H}$ & 7.44185 & -0.48701 & -1.67637 \\
\hline $\mathrm{H}$ & 8.80386 & -1.15095 & -0.59775 \\
\hline $\mathrm{H}$ & 6.65482 & -2.71971 & 1.72902 \\
\hline $\mathrm{H}$ & 6.91418 & -1.11216 & 2.38692 \\
\hline $\mathrm{H}$ & 8.26946 & -1.98227 & 1.63455 \\
\hline $\mathrm{O}$ & -4.81541 & -1.40172 & 0.70464 \\
\hline $\mathrm{C}$ & -5.66434 & -0.7735 & -0.09603 \\
\hline $\mathrm{O}$ & -5.55344 & 0.41331 & -0.41423 \\
\hline $\mathrm{C}$ & -6.77677 & -1.64708 & -0.58007 \\
\hline $\mathrm{C}$ & -7.68291 & -1.10093 & -1.40033 \\
\hline $\mathrm{C}$ & -6.81104 & -3.08094 & -0.11885 \\
\hline $\mathrm{H}$ & -4.09389 & -0.76939 & 1.00285 \\
\hline $\mathrm{H}$ & -7.60587 & -0.06073 & -1.69857 \\
\hline $\mathrm{H}$ & -8.51623 & -1.67957 & -1.78869 \\
\hline $\mathrm{H}$ & -6.87012 & -3.14431 & 0.97354 \\
\hline $\mathrm{H}$ & -5.89943 & -3.61349 & -0.4125 \\
\hline $\mathrm{H}$ & -7.67236 & -3.60308 & -0.54547 \\
\hline
\end{tabular}

Compound $=$ HVA-(VPY $)_{2}$

$\begin{array}{cccc}\mathrm{C} & 2.70117 & 5.47702 & 2.37304 \\ \mathrm{~N} & 2.6786 & 4.5724 & 1.38168 \\ \mathrm{C} & 3.68404 & 3.68784 & 1.31891 \\ \mathrm{C} & 4.73396 & 3.67823 & 2.23348 \\ \mathrm{C} & 4.76806 & 4.6183 & 3.27477 \\ \mathrm{C} & 3.70724 & 5.53962 & 3.3278 \\ \mathrm{C} & 5.88201 & 4.59665 & 4.23577 \\ \mathrm{C} & 6.04601 & 5.40096 & 5.29278 \\ \mathrm{H} & 1.87229 & 6.18088 & 2.39711 \\ \mathrm{H} & 3.62112 & 2.97502 & 0.50047 \\ \mathrm{H} & 5.52463 & 2.93928 & 2.13634 \\ \mathrm{H} & 3.66104 & 6.30031 & 4.10025\end{array}$




$\begin{array}{cccc}\mathrm{H} & 6.63518 & 3.83392 & 4.04156 \\ \mathrm{H} & 6.90999 & 5.29862 & 5.942 \\ \mathrm{H} & 5.33884 & 6.183 & 5.55562 \\ \mathrm{O} & 0.7598 & 4.2852 & 0.61309 \\ \mathrm{C} & 1.07992 & 3.24975 & 1.38777 \\ \mathrm{O} & 2.08008 & 2.56229 & 1.23695 \\ \mathrm{C} & 0.05016 & 2.98541 & 2.47663 \\ \mathrm{C} & 0.87911 & 1.83849 & 2.10898 \\ \mathrm{C} & 2.25787 & 2.02557 & 2.02189 \\ \mathrm{C} & 3.10328 & 0.96122 & 1.69944 \\ \mathrm{C} & 2.59255 & 0.31228 & 1.45079 \\ \mathrm{C} & 1.19342 & 0.50258 & 1.53456 \\ \mathrm{C} & 0.35476 & 0.55907 & 1.85931 \\ \mathrm{O} & 0.76562 & 1.78575 & 1.27441 \\ \mathrm{C} & 1.99972 & 0.62899 & 1.14225 \\ \mathrm{O} & 3.44919 & 1.30905 & 1.13201 \\ \mathrm{H} & 1.45448 & 4.38837 & 0.10908 \\ \mathrm{H} & 0.60786 & 2.74582 & 3.39006 \\ \mathrm{H} & 0.53137 & 3.89316 & 2.65554 \\ \mathrm{H} & 2.68319 & 3.0089 & 2.20652 \\ \mathrm{H} & 4.17793 & 1.0999 & 1.62819 \\ \mathrm{H} & 0.71937 & 0.41978 & 1.90536 \\ \mathrm{H} & 0.7479 & 3.04364 & 0.84323 \\ \mathrm{H} & 1.06316 & 1.3474 & 0.37361 \\ \mathrm{H} & 1.15794 & 1.83185 & 2.09026 \\ \mathrm{H} & 2.99632 & 2.17931 & 1.00617 \\ \mathrm{C} & 3.85042 & 4.64763 & 0.05831 \\ \mathrm{~N} & 2.94359 & 4.05597 & 0.73375 \\ \mathrm{C} & 2.07937 & 4.84814 & 1.37869 \\ \mathrm{C} & 2.08343 & 6.23564 & 1.26096 \\ \mathrm{C} & 3.02551 & 6.86291 & 0.43079 \\ \mathrm{C} & 3.9299 & 6.02292 & 0.2414 \\ \mathrm{C} & 3.02257 & 8.32933 & 0.30848 \\ \mathrm{C} & 3.82734 & 9.07217 & 0.46069 \\ \mathrm{H} & 4.54408 & 3.98055 & 0.5651 \\ \mathrm{H} & 1.35929 & 4.33985 & 2.01472 \\ \mathrm{H} & 1.35771 & 6.82779 & 1.81204 \\ \mathrm{H} & 4.69208 & 6.42954 & 0.89822 \\ \mathrm{H} & 2.27454 & 8.83513 & 0.918 \\ \mathrm{H} & 3.74017 & 10.15409 & 0.47671 \\ \mathrm{H} & 4.59496 & 8.64316 & 1.09881 \\ & & & \end{array}$

$\begin{array}{llll}\mathrm{H} & -5.19419 & -2.28147 & -0.1676 \\ \mathrm{H} & -5.7851 & -1.30633 & -1.5036 \\ \mathrm{H} & -6.82015 & -1.56828 & -0.08216 \\ \mathrm{H} & -5.44093 & 1.48765 & 1.54238 \\ \mathrm{H} & -6.92194 & 0.37406 & 1.39049\end{array}$

Compound $=$ IVMA-MAA

$\begin{array}{llcc}\mathrm{C} & 1.87086 & -0.80559 & -0.40766 \\ \mathrm{C} & 1.98254 & 0.58788 & -0.53705 \\ \mathrm{C} & 2.93989 & 1.27439 & 0.20525 \\ \mathrm{C} & 3.78667 & 0.58531 & 1.08402 \\ \mathrm{C} & 3.67124 & -0.79341 & 1.21517 \\ \mathrm{C} & 2.70644 & -1.49451 & 0.46073 \\ \mathrm{C} & 1.07692 & 1.35165 & -1.51391 \\ \mathrm{C} & -0.3855 & 0.99483 & -1.24044 \\ \mathrm{O} & -0.97518 & 1.85215 & -0.42683 \\ \mathrm{O} & 4.42587 & -1.60189 & 2.03241 \\ \mathrm{O} & 2.59547 & -2.84687 & 0.58683 \\ \mathrm{O} & -0.92007 & -0.00442 & -1.71482 \\ \mathrm{O} & 1.27326 & 2.75057 & -1.48893 \\ \mathrm{C} & 5.43688 & -0.99656 & 2.82347 \\ \mathrm{H} & 1.13537 & -1.3671 & -0.97477 \\ \mathrm{H} & 3.04084 & 2.34676 & 0.0852 \\ \mathrm{H} & 4.52875 & 1.1348 & 1.6531 \\ \mathrm{H} & 1.29132 & 1.02742 & -2.5377 \\ \mathrm{H} & -1.92915 & 1.57263 & -0.24461 \\ \mathrm{H} & 3.26529 & -3.12548 & 1.23565 \\ \mathrm{H} & 0.78664 & 3.0894 & -0.71826 \\ \mathrm{H} & 5.01051 & -0.26752 & 3.52449 \\ \mathrm{H} & 5.9089 & -1.80561 & 3.38368 \\ \mathrm{H} & 6.18805 & -0.49961 & 2.19624 \\ \mathrm{O} & -3.47509 & 1.12477 & 0.06158 \\ \mathrm{C} & -4.03445 & 0.11297 & -0.37351 \\ \mathrm{O} & -3.45012 & -0.76095 & -1.17858 \\ \mathrm{C} & -5.44601 & -0.2437 & -0.03833 \\ \mathrm{C} & -6.0221 & -1.50487 & -0.62777 \\ \mathrm{C} & -6.11926 & 0.58303 & 0.77142 \\ \mathrm{H} & -2.50767 & -0.47755 & -1.3718 \\ \mathrm{H} & -5.43817 & -2.38319 & -0.33073 \\ \mathrm{H} & -6.00403 & -1.47467 & -1.72302 \\ \mathrm{H} & -7.05603 & -1.64615 & -0.30005 \\ \mathrm{H} & -5.64998 & 1.47794 & 1.16599 \\ \mathrm{H} & -7.14846 & 0.3826 & 1.05504 \\ & & & \\ & & & \end{array}$

Compound $=$ MHPE-MAA

$-0.35832$ 0.42361

1.2227

1.24163

0.4447

$-1.24956$

$-0.97158$

$-0.10761$

2.01834

0.55276

$-0.20394$

$-1.48679$

$-1.11816$

$-0.9575$

0.39478

1.83521

$-2.30185$

0.07544

1.87924

0.03139

0.07376

$-1.28049$

$-0.30641$

0.38498

$-0.09563$

$-0.95598$

0.23969

$-0.41157$

1.10444

$-1.14853$

$\begin{array}{lccc}\mathrm{C} & -5.33890 & 0.679364 & 1.308496 \\ \mathrm{C} & -6.26258 & -0.444778 & 0.915859 \\ \mathrm{C} & -5.62292 & 1.593588 & 2.244845 \\ \mathrm{H} & -2.94717 & -0.022462 & -0.694738 \\ \mathrm{H} & -5.79086 & -1.421007 & 1.075213 \\ \mathrm{H} & -6.52400 & -0.392319 & -0.147135 \\ \mathrm{H} & -7.18505 & -0.405661 & 1.501991 \\ \mathrm{H} & -4.91619 & 2.380880 & 2.485243 \\ \mathrm{H} & -6.56341 & 1.572737 & 2.78770\end{array}$


Table S2. Absolute energies calculated with BS I and BS II (a.u.) and ZPVE obtained with BS I (kcal/mol) for isolated reactants and all the complexes located.

\begin{tabular}{|c|c|c|c|}
\hline Structures & $\begin{array}{l}\text { Absolute Energies } \\
\text { BS I = 6-31G* }\end{array}$ & $\begin{array}{c}\text { ZPVE -BS I } \\
\text { (kcal/mol) }\end{array}$ & $\begin{array}{l}\text { Absolute Energies } \\
\text { BS II = 6-311+G** }\end{array}$ \\
\hline \multicolumn{4}{|c|}{ Isolated reactants } \\
\hline HVA & -649.8713847 & 113.88905 & -650.0744244 \\
\hline MAA & -306.4797422 & 60.20577 & -306.5811889 \\
\hline VPY & -325.6849792 & 76.55302 & -325.7712609 \\
\hline \multicolumn{4}{|c|}{ The most stable complexes } \\
\hline HVA-MAA & -956.3826476 & 175.16594 & -956.6811063 \\
\hline HVA-VPY & -975.5767273 & 191.2641 & -975.8629947 \\
\hline HVA-(MAA) $)_{2}$ & -1262.880510 & 236.32032 & -1263.2749713 \\
\hline $\mathrm{HVA}(\mathrm{VPY})_{2}$ & -1301.273527 & 268.55184 & -1301.6426443 \\
\hline \multicolumn{4}{|c|}{ The least stable complexes } \\
\hline HVA-MAA2 & -956.3752605 & 175.01344 & -956.6735448 \\
\hline HVA-MAA3 & -956.3690534 & 174.97074 & -956.6680247 \\
\hline HVA-MAA4 & -956.3679005 & 175.02601 & -956.6666285 \\
\hline HVA-MAA5 & -956.3663178 & 175.01611 & -956.6658503 \\
\hline HVA-MAA6 & -956.3650853 & 174.83391 & -956.6662583 \\
\hline HVA-MAA7 & -956.3633923 & 175.02257 & -956.6616753 \\
\hline HVA-MAA8 & -956.3610982 & 174.99499 & -956.6611348 \\
\hline HVA-MAA9 & -956.3590975 & 174.54760 & -956.6578045 \\
\hline HVA-MAA10 & -956.3536127 & 174.85649 & -956.653464 \\
\hline HVA-VPY2 & -975.5681498 & 191.05660 & -975.8544686 \\
\hline HVA-VPY3 & -975.5674837 & 190.77025 & -975.8537698 \\
\hline
\end{tabular}


Figure S1: B3LYP/6-31G* geometries of the least stable pre-polymerization complexes formed between HVA and the functional monomers MAA and VPY.

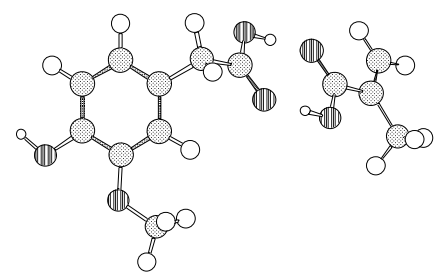

HVA-MAA2

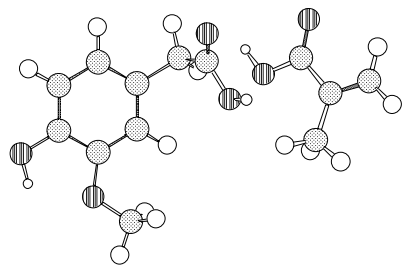

HVA-MAA5

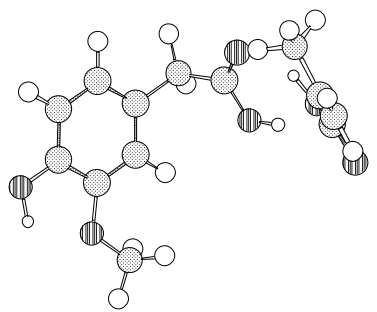

HVA-MAA8

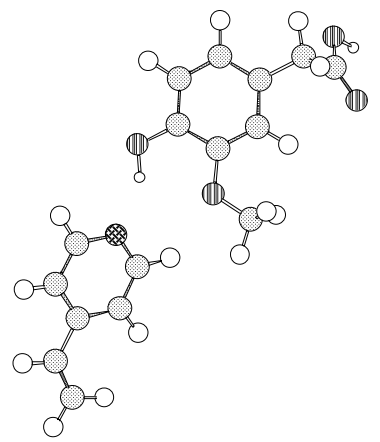

HVA-VPY2

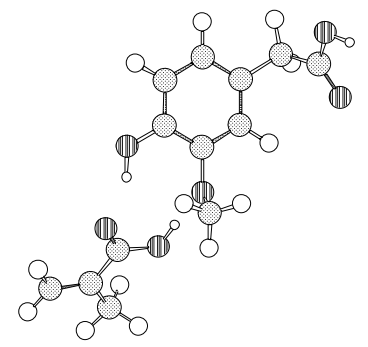

HVA-MAA3

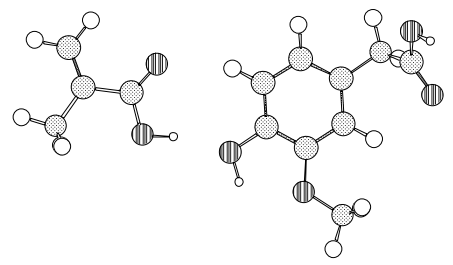

HVA-MAA6

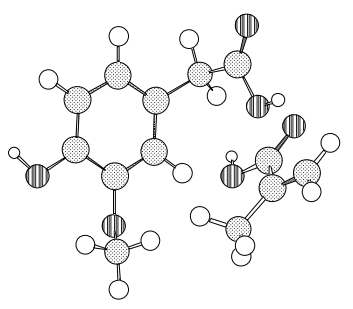

HVA-MAA9

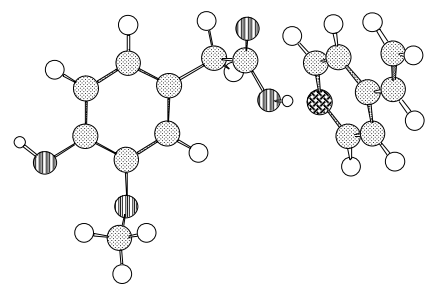

HVA-VPY3
HVA-MAA10

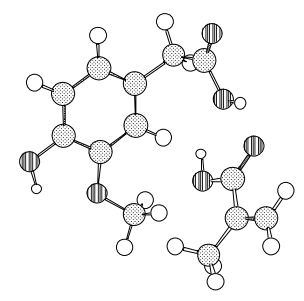

HVA-MAA4

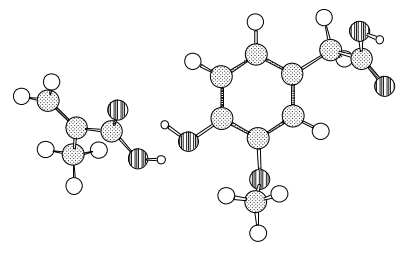

HVA-MAA7

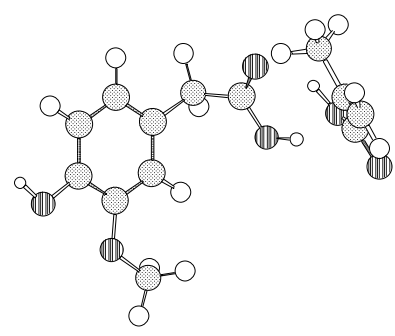

(IIII) $\mathrm{O}$

誉叕 $\mathrm{N}$

C

H 